\title{
The impact of diabetes mellitus on cognitive decline in the oldest of the old: a prospective population-based study
}

\author{
E. van den Berg • A. J. M. de Craen • G. J. Biessels • \\ J. Gussekloo • R. G. J. Westendorp
}

Received: 19 January 2006 / Accepted: 5 May 2006 / Published online: 28 June 2006

(C) Springer-Verlag 2006

\begin{abstract}
Aims/hypothesis Diabetes mellitus is a risk factor for the development of cognitive impairment and dementia in the general population up to 75 years of age. As part of the Leiden 85-plus Study we studied the effects of diabetes on cognition in the oldest old.

Subjects and methods The Leiden 85-plus Study is a prospective population-based study of 599 persons from age 85 onward. Cognitive function was assessed each year from ages 85 to 90 by means of four neuropsychological tests. The presence of diabetes and vascular disease was recorded at baseline, $\mathrm{HbA}_{1 \mathrm{c}}$ was assessed by means of a blood sample at ages 85 and 90 . The cross-sectional and prospective associations between diabetes and cognitive function were analysed with linear mixed models, adjusted for sex and level of education.

Results At age 85, diabetes was associated with a lower level of cognitive functioning on the Letter Digit Coding
\end{abstract}

E. van den Berg · G. J. Biessels

Department of Neurology, Rudolf Magnus Institute

of Neuroscience, University Medical Center,

Utrecht, The Netherlands

\author{
A. J. M. de Craen · R. G. J. Westendorp \\ Department of Gerontology and Geriatrics, \\ Leiden University Medical Center, \\ Leiden, The Netherlands \\ J. Gussekloo \\ Department of Public Health and Primary Care, \\ Leiden University Medical Center, \\ Leiden, The Netherlands \\ G. J. Biessels $(\bowtie)$ \\ Department of Neurology, G03.228, University Medical Center, \\ P.O. Box 85500, 3508 GA Utrecht, The Netherlands \\ e-mail: g.j.biessels@umcutrecht.nl
}

test and the Stroop Test. Diabetes was not associated with accelerated cognitive decline during follow-up. Within the group of diabetic patients, macrovascular disease was the most important determinant of cognitive dysfunction.

Conclusions/interpretation The association between diabetes and accelerated cognitive decline, which has been documented previously in patients up to 75 years of age, may be less evident after age 85 . This suggests that the damage occurs in previous years and that therapies aimed at preventing cognitive decline and dementia should focus on the younger old.

Keywords Diabetes mellitus · Cognitive decline · Oldest old

\author{
Abbreviations \\ MMSE Mini-Mental State Examination \\ GDS-15 15-item Geriatric Depression Scale
}

\section{Introduction}

Type 2 diabetes mellitus is a common metabolic disease with prevalence estimates around $10 \%$ in persons over 65 years of age [1]. There are several complications, such as nephropathy, neuropathy, retinopathy and cardiovascular disease [2]. In recent years it has been shown that type 2 diabetes can also affect the brain. Cross-sectional neuropsychological studies show moderate degrees of cognitive impairment in patients with type 2 diabetes, particularly in verbal memory, information-processing speed and, less consistently, executive functions [3-5]. Longitudinal studies show accelerated decline in cognitive functions $[6,7]$. In addition, a two-fold increased risk of developing Alzheimer's disease or vascular dementia has been reported 
[7-9]. It has been suggested that the effects of diabetes on cognition are most pronounced in the elderly [10].

In the general population, studies on other risk factors for accelerated cognitive decline, such as hypertension and obesity, indicate that the impact of these factors may be age-dependent. For example, although midlife hypertension is a risk factor for late-life cognitive impairment and dementia, the relation between blood pressure and cognitive decline in persons of advanced age is less clear [11]. In order to develop strategies aimed at the treatment of these potentially modifiable risk factors for cognitive decline and dementia, further insight into the temporal relation of these factors with cognition in different age groups is required. For diabetes, this information is largely lacking for persons of advanced age.

The aim of the present study, conducted as part of the Leiden 85-plus Study [12], was to examine the impact of diabetes mellitus on cognitive decline over time in the oldest of the old. We were in the unique position of being able to study this association both cross-sectionally and longitudinally in a population of 85 -year-old people participating in the Leiden 85-plus Study. We hypothesised that diabetes is associated with a lower level of cognitive functioning at age 85 and an accelerated cognitive decline from 85 to 90 years of age. In addition, to identify determinants of cognitive dysfunction in diabetic patients, we assessed the effects of disease variables such as chronic exposure to elevated glucose levels and macrovascular disease.

\section{Subjects and methods}

\section{Subjects}

The Leiden 85-plus study is a prospective population-based study of all 85-year-old inhabitants of Leiden. Between 1 September 1997 and 1 September 1999, 599 participants were enrolled (response rate $87 \%$ ). The non-responders were generally healthier and had poorer mood, but had mortality similar to that of the responders [12]. Participants were visited annually from the age of 85 to 90 years at their home for face-to-face interviews. The medical ethics committee of the Leiden University Medical Center approved the study and all subjects gave informed consent to participation. For the present analysis, we included the 596 (99\%) participants for whom diabetes status was determined. Blood samples were obtained from 562 participants.

\section{Diabetes mellitus}

At baseline, participants who had a history of diabetes mellitus were identified through their general practitioner's medical record and medication use. These participants were classified as having 'known diabetes' $(n=86)$. Eight participants used insulin, 45 used only oral antidiabetic medication, and 33 were treated with diet only. The nonfasting plasma glucose concentration was determined in all participants at baseline and again annually. Levels of $\mathrm{HbA}_{1 \mathrm{c}}$ were determined at ages 85 and 90. Participants without a history of diabetes but with a non-fasting plasma glucose concentration greater than $11 \mathrm{mmol} / \mathrm{l}$ at baseline were classified as having 'newly diagnosed diabetes' $(n=10)$. These patients were advised to consult their general practitioner for further evaluation and to consider treatment. No specific information was obtained on the type of diabetes ( 1 or 2$)$. Given the advanced age of the participants, however, the vast majority of the patients would have had type 2 diabetes mellitus. In sum, of the 596 participants, $96(16 \%)$ were classified as having diabetes.

Cognitive function and depressive symptoms

Global cognitive function was assessed with the MiniMental State Examination (MMSE) [13]. MMSE scores range from 0 to 30 , lower scores indicating impaired cognitive functioning. All participants performed four neuropsychological tests that are reliable and sensitive in the detection of small differences in elderly persons [14]. Attention was measured using the third chart of the 40-item Stroop Test. The time needed to name the ink colour of incongruously printed names of colours is taken as a measure of attention, higher scores indicating poorer attention. Information-processing speed was assessed with the Letter Digit Coding Test; the outcome was the total number of correct digits according to a code key in $60 \mathrm{~s}$, lower scores indicating slower speed. Memory (immediate and delayed recall) was measured with the 12 Word Learning Test. In this test, 12 pictures are presented to the participant, who is then asked to recall them. This procedure is rehearsed three times. The total number of words that are correctly recalled immediately after each procedure is taken as a measure of immediate recall. Delayed recall is measured as the number of correct words that are correctly recalled after $20 \mathrm{~min}$. Lower scores indicate worse memory. Parallel versions for the coding and memory tests, using identical procedures but with different items, were available to prevent learning effects. Depressive symptoms were assessed annually with the 15 -item Geriatric Depression Scale (GDS-15), a questionnaire specially developed as a screening instrument for depressive symptoms in elderly populations [15]. Participants with a GDS15 score of 4 or higher were considered to have clinically relevant depressive symptoms [16]. The Stroop Test, Letter Digit Coding Test, 12 Word Learning Test and GDS-15 were not assessed in participants with an MMSE score of 
18 points or lower, because of the lack of reliability and validity in subjects with severe cognitive impairment.

Vascular disease

The presence of vascular disease was assessed from the history taken from the treating physician and the ECG. The following vascular pathologies were recorded with a standardised questionnaire: stroke; myocardial infarction; intermittent claudication; and arterial surgery. Stroke, arterial surgery and intermittent claudication were considered present when there was a positive medical history. Myocardial infarction was considered present when there was a positive medical history or when the ECG revealed myocardial infarction (Minnesota codes 1-1, 1-2 and 1-3) [17]. Hypertension was defined as a systolic pressure greater than $160 \mathrm{~mm} \mathrm{Hg}$, a diastolic pressure greater than $95 \mathrm{~mm} \mathrm{Hg}$, or the use of antihypertensive medication.

\section{Statistical analysis}

For the baseline analysis, comparisons between participants with and without diabetes for continuous parametric data were performed with the $t$-test; proportions were analysed with the $\chi^{2}$ test. Since mortality will be high in a population of the oldest old and may be expected to be even higher among diabetic patients, Cox regression analysis was performed.

The association between diabetes and cognitive function at baseline and during follow-up was analysed with linear mixed models. Mixed models use all available data during follow-up, can properly account for correlation between repeated measurements, and can handle missing data more appropriately than traditional models. They also allow the use of time-independent and time-dependent covariates [18]. Cognitive function was entered in the models using the test scores obtained annually. Models studying the relation between diabetes and cognitive function included diabetes status $(\mathrm{Y} / \mathrm{N})$ at baseline, time, and an interaction between diabetes status and time. The estimate of the effect of diabetes status reflects the cross-sectional effect of diabetes on cognitive function, and is presented as the cross-sectional effect. The estimate of the effect of time reflects the annual change in cognitive function, and is presented as change over time. The estimate of interaction of diabetes status with time reflects the additional annual effect of diabetes on cognitive function and is presented as the additional annual effect. All models were adjusted for sex and level of education.

The relation between $\mathrm{HbA}_{1 \mathrm{c}}$ and cognition across the whole population was investigated in a similar way, adjusting for sex, level of education and diabetes status $(\mathrm{Y} / \mathrm{N}) \cdot \mathrm{HbA}_{1 \mathrm{c}}$ was entered in the analytical models as a continuous variable. We standardised the estimates per SD increase in $\mathrm{HbA}_{1 \mathrm{c}}$ level by using the formula: (individual serum level - mean serum level in the population)/SD in the population.

Within the group of diabetic patients, linear mixed models (adjusted for sex and level of education) were used to examine the effects of the following variables on cognitive function: $\mathrm{HbA}_{1 \mathrm{c}}$ level (per SD increase), cardiovascular disease (myocardial infarction, arterial surgery and intermittent claudication), stroke and hypertension. In a post hoc analysis we repeated the analysis of the association between diabetes and cognition while adjusting for the presence of macrovascular disease (myocardial infarction, arterial surgery, intermittent claudication, or stroke) and depressive symptoms. Since only eight participants used insulin, the role of insulin treatment could not be examined reliably.

Dementia was not formally assessed as an outcome measure. In secondary analyses, we calculated odds ratios for MMSE scores of 18 or less (as a surrogate measure of dementia) and 23 or less (surrogate measure of cognitive impairment) for the diabetic relative to the non-diabetic participants.

\section{Results}

Table 1 summarises the characteristics of the diabetic $(n=96)$ and the non-diabetic participants $(n=500)$ at age 85. Participants with diabetes had a significantly lower educational level and higher glucose and $\mathrm{HbA}_{1 \mathrm{c}}$ levels. No differences in the history of vascular disease were found between the diabetic and non-diabetic participants. The diabetic patients had an increased risk of mortality during follow-up (hazard ratio 1.5 (95\% CI 1.1-2.0)). From the 596 patients who were included at baseline, 44 were lost to follow-up over the 5 years of the study. During follow-up 263 participants died. Common causes of death were cardiovascular disease (42\%), infectious diseases $(18 \%)$ and cancer (17\%); these proportions were similar in the diabetic and non-diabetic groups.

The effect of diabetes on annual assessments of cognitive function is shown in Fig. 1 and Table 2. Crosssectionally, the linear mixed models showed that diabetes was associated with poorer performance on the Letter Digit Coding Test and the Stroop Test (cross-sectional effect). During follow-up, there was a significant decline in all but one measure of cognitive function (change over time). Diabetes was not associated with an accelerated decline in any of the cognitive tests (additional annual effect). Both exclusion of the participants who developed diabetes during follow-up ( $n=17)$ and repeating our analyses only with the participants who survived the follow-up $(n=289)$ did not 
Table 1 Baseline characteristics of the participants

No diabetes $(n=500)$

$$
174(35 \%)
$$

$313(63 \%)$

$86(17 \%)$

$84(17 \%)$

Institutionalised $^{\mathrm{b}}$

Diabetes characteristics

Antidiabetic medication

Oral antidiabetic medication

Insulin

Glucose $^{\mathrm{c}}$

$\mathrm{HbA}_{1 \mathrm{c}} \mathrm{c}^{\mathrm{c}}$

Vascular disease

Myocardial infarction

Arterial surgery

Intermittent claudication

History of stroke

Hypertension

Depressive symptoms

GDS-15

GDS- 15 score $\geq 4$

$6.3 \pm 1.4$

$128(26 \%)$

$32(6 \%)$

$30(6 \%)$

$50(10 \%)$

2 (1-3)
Diabetes $(n=96)$

$p$

27 (28\%)

$71(74 \%)$

0.21

$17(18 \%)$

0.02

$24(25 \%)$

0.92

0.06

$53(55 \%)$

45

8

$10.4 \pm 4.4$

$<0.001$

$7.2 \pm 1.7$

$<0.001$

27 (28\%)

0.61

$8(8 \%)$

0.50

$7(8 \%)$

0.60

$12(13 \%)$

0.47

$70(76 \%)$

0.22

$2(1-3)$

0.19

$36(38 \%)$

Data are $n(\%)$, mean \pm SD or median (interquartile range)

${ }^{\text {a }}$ State pension only

${ }^{\mathrm{b}}$ Includes homes for the elderly and nursing homes; fewer than $20 \%$ were admitted for psychogeriatric reasons; institutionalised participants were included in the neuropsychological assessment

${ }^{\mathrm{c}}$ Blood samples were obtained from 562 participants

a

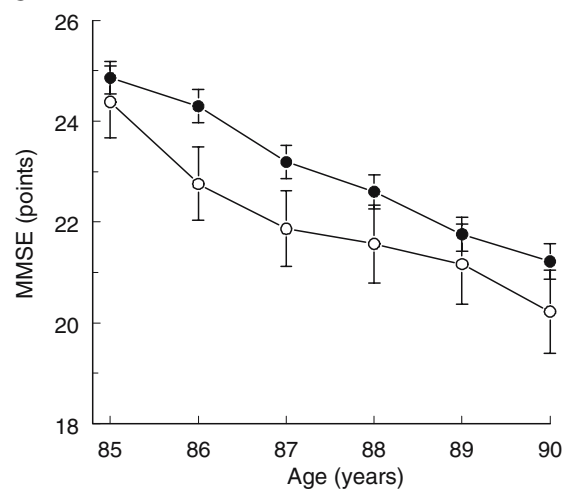

d

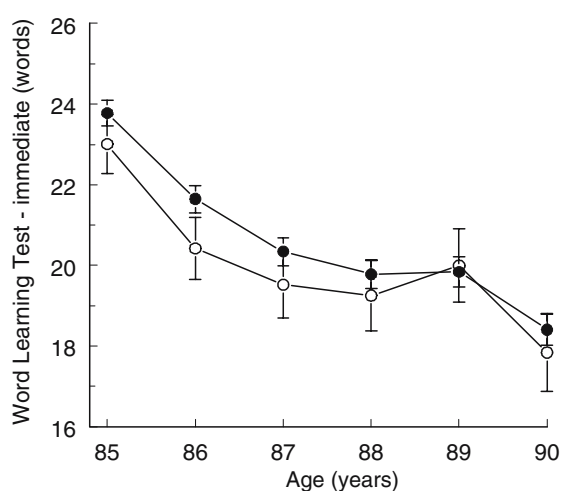

b

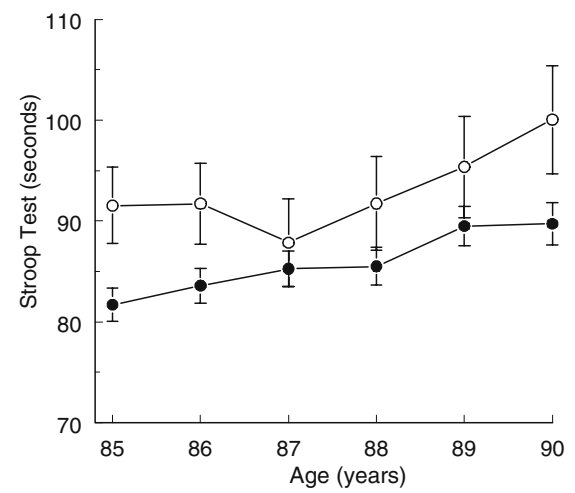

e

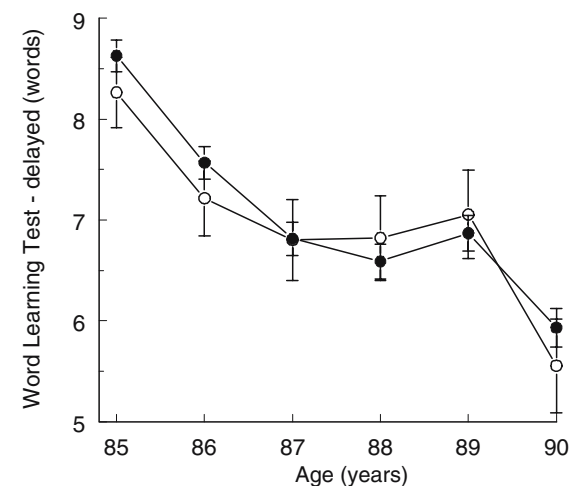

C

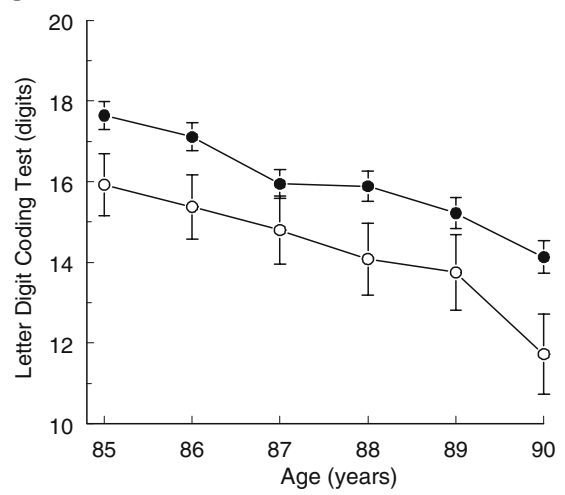

Fig. 1 a-e Cognitive function in diabetic (open circles; $n=96$ at baseline) and non-diabetic (filled circles; $n=500$ at baseline) participants from age 85 to age 90 , estimated from linear mixed models adjusted for sex and level of education. Data are mean and SE 
Table 2 Effects of diabetes and $\mathrm{HbA}_{1 \mathrm{c}}$ on cognitive function from age 85 to 90 years

\begin{tabular}{|c|c|c|c|c|c|c|}
\hline \multirow[t]{2}{*}{ Model } & \multicolumn{2}{|c|}{ Cross-sectional effect ${ }^{\mathrm{a}}$} & \multicolumn{2}{|c|}{ Change over time ${ }^{b}$} & \multicolumn{2}{|c|}{ Additional annual effect ${ }^{\mathrm{c}}$} \\
\hline & Estimate (SE) & $p$ & Estimate (SE) & $p$ & Estimate (SE) & $p$ \\
\hline \multicolumn{7}{|l|}{ Diabetes (reference: diabetes status $=$ no) } \\
\hline MMSE (points) & $-0.93(0.74)$ & 0.21 & $-0.78(0.09)$ & $<0.001$ & $-0.02(0.10)$ & 0.82 \\
\hline Stroop Test $(\mathrm{s})^{\mathrm{d}}$ & $8.38(3.78)$ & 0.03 & $1.23(0.82)$ & 0.13 & $-0.43(0.87)$ & 0.63 \\
\hline Letter Digit Coding Test (digits) & $-1.60(0.79)$ & 0.045 & $-0.71(0.13)$ & $<0.001$ & $-0.05(0.14)$ & 0.70 \\
\hline Word Learning Test_-immediate (words) & $-0.89(0.75)$ & 0.24 & $-0.86(0.14)$ & $<0.001$ & $0.14(0.15)$ & 0.35 \\
\hline Word Learning Test—-delayed (words) & $-0.27(0.36)$ & 0.45 & $-0.41(0.07)$ & $<0.001$ & $0.07(0.07)$ & 0.32 \\
\hline \multicolumn{7}{|l|}{$\mathrm{HbA}_{1 \mathrm{c}}$ (per SD increase) } \\
\hline MMSE (points) & $0.49(0.27)$ & 0.07 & $-0.53(0.06)$ & $<0.001$ & $0.04(0.07)$ & 0.54 \\
\hline Stroop Test $(\mathrm{s})^{\mathrm{d}}$ & $-0.84(1.55)$ & 0.59 & $1.31(0.35)$ & $<0.001$ & $0.28(0.41)$ & 0.49 \\
\hline Letter Digit Coding Test (digits) & $-0.01(0.31)$ & 0.89 & $-0.56(0.07)$ & $<0.001$ & $0.09(0.08)$ & 0.29 \\
\hline Word Learning Test-immediate (words) & $0.06(0.32)$ & 0.84 & $-0.83(0.08)$ & $<0.001$ & $0.08(0.09)$ & 0.33 \\
\hline Word Learning Test-delayed (words) & $-0.06(0.15)$ & 0.69 & $-0.43(0.04)$ & $<0.001$ & $-0.01(0.04)$ & 0.73 \\
\hline
\end{tabular}

Model 'Diabetes' $(n=596)$ is adjusted for sex and educational level; model ' $\mathrm{HbA}_{1 \mathrm{c}}$ ' $(n=562)$ is adjusted for sex, educational level and the presence of diabetes

${ }^{a}$ The estimate for the cross-sectional effect shows the mean difference between participants with and without diabetes or per SD increase in $\mathrm{HbA} \mathrm{A}_{1 \mathrm{c}}$

${ }^{\mathrm{b}}$ The estimate for change over time shows the annual change per test across the whole population

${ }^{\mathrm{c}}$ The estimate for the additional effect shows the annual change that is attributable to diabetes status, or each $\mathrm{SD}$ increase in $\mathrm{HbA}_{1 \mathrm{c}}$ across the whole population

${ }^{\mathrm{d}}$ Higher scores reflect worse performance

notably alter the results. Furthermore, exclusion of the participants with newly diagnosed diabetes $(n=10)$ did not alter the results. Across the whole population (diabetic and non-diabetic participants), there was no cross-sectional association between the level of $\mathrm{HbA}_{1 \mathrm{c}}$ and cognitive dysfunction (Table 2). $\mathrm{HbA}_{1 \mathrm{c}}$ was also not associated with accelerated cognitive decline during follow-up. Excluding participants who used antidiabetic medication $(n=53)$ did not alter the results. At age 85 the odds ratios for an MMSE score of 18 or less and 23 or less in the diabetic group were 0.9 (95\% CI $0.5-1.7)$ and $1.3(0.8-2.1)$, respectively, relative to the non-diabetic group. At age 90, including only subjects with an MMSE greater than 18 (or greater than 23) at age 85, the odds ratio was $1.3(0.5-3.0)$ for an MMSE score of 18 or less and 1.2 (0.5-2.9) for an MMSE score of 23 or less.

Within the group of diabetic patients, lower baseline cognitive function was associated with a history of stroke (Table 3). There was no clear relation between cognition and hypertension. If anything, diabetic patients with hypertension tended to perform slightly better across different tests. Analyses with systolic and diastolic blood pressures as continuous variables confirm these results (e.g. cross-sectional effect systolic blood pressure, MMSE 0.7 [0.0-1.5] per $10 \mathrm{~mm} \mathrm{Hg}$; diastolic blood pressure, 1.1 $[-0.3-2.6]$ per $10 \mathrm{~mm} \mathrm{Hg}$ ). The longitudinal analysis showed an accelerated cognitive decline for diabetic patients with a history of arterial surgery and intermittent claudication. A history of stroke appeared to be associated with decelerated cognitive decline in the longitudinal analysis. However, taking into account the large effect of stroke on the MMSE score, this probably reflects a ceiling effect. A higher $\mathrm{HbA}_{1 \mathrm{c}}$ level was related to a decelerated decline on the memory tests. The associations between lower cognitive function and macrovascular disease were not specific for the diabetic group. Similar associations have been shown for the whole Leiden 85-plus population [19]. The repeated analysis of the association between diabetes and cognition while adjusting for the presence of macrovascular disease (myocardial infarction, arterial surgery, intermittent claudication, or stroke) showed a 5-10\% reduction in the estimates of the difference between diabetic and non-diabetic participants, as presented in Table 2 (MMSE $-0.82, p=0.27$; Stroop Test 7.77, $p=0.04$; Letter Digit Coding Test $-1.51, p=0.06$; Word Learning Test, Immediate $-0.85, p=0.26$; Word Learning Test, Delayed $-0.26, p=0.48$ ). Repeated analysis of the association between diabetes and cognition while adjusting for depressive symptoms did not alter the results notably.

\section{Discussion}

The results of the present study show that at age 85 diabetes is associated with a lower level of cognitive functioning on the Letter Digit Coding Test and the Stroop Test. This is in line with previous cross-sectional studies of cognitive function in diabetes, which show moderate impairments in information-processing speed and executive functions [3-5]. Memory function, which is usually also found to be 


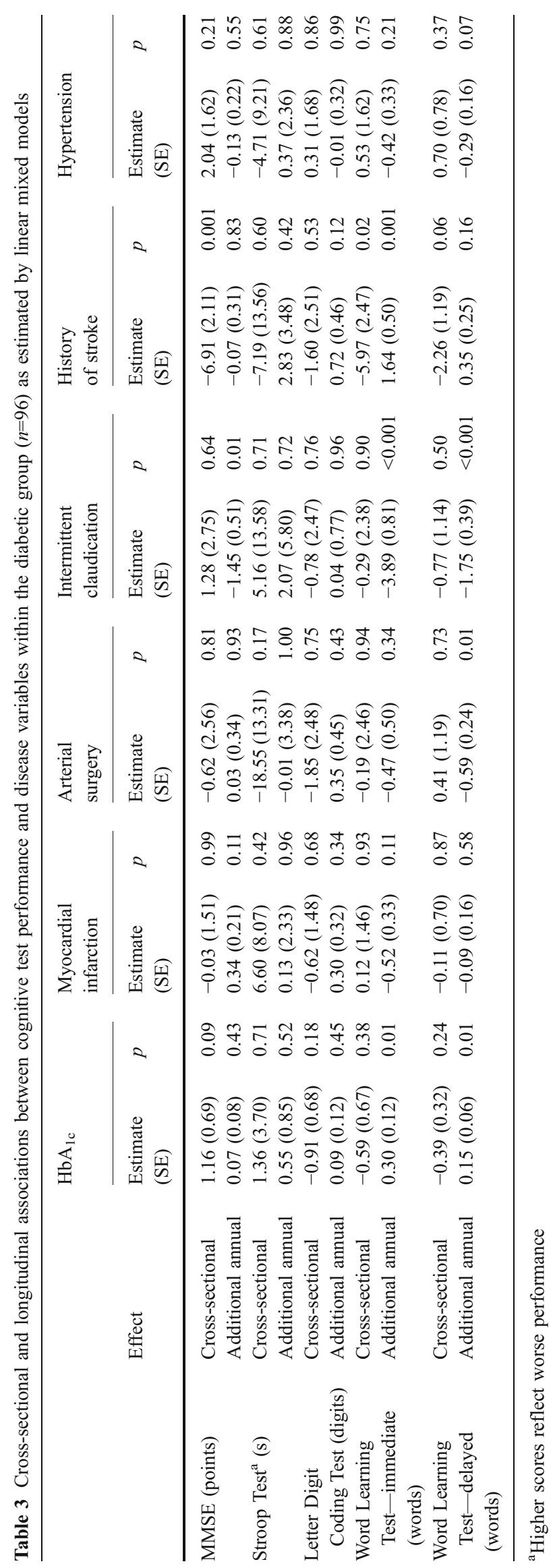


impaired, did not differ between the diabetic and nondiabetic participants. The impairments found in the present study involved tests in which time or speed is essential. It has been hypothesised that the cognitive performance of diabetic persons is particularly affected when the cognitive task at hand requires speed of response and when the individual has to respond within a fixed time limit [20].

The results also show that although the population as a whole shows a marked decline in all cognitive functions from age 85 to age 90 , diabetes was not associated with accelerated cognitive decline. To date, a limited number of studies have examined the effect of diabetes on cognition longitudinally (for a review, see $[6,7]$ ). The majority of these studies address the development of dementia and usually show an increased incidence of both Alzheimer's disease and vascular dementia in older (mean age 69-77) diabetes patients [21-23]. There are only seven populationbased longitudinal studies that have examined cognitive function by means of a cognitive test battery [24-30]. In five of these the difference in cognitive performance between diabetic and non-diabetic participants was examined both at baseline and prospectively [24-27, 29]. In all but one [24] of these studies, the mean age of the population at baseline was between 65 and 75 years. Two studies specifically included participants who were cognitively intact (e.g. MMSE score $>26$ ) at baseline, resulting in no significant differences in baseline cognitive function [24, 27]. Out of the remaining three studies, two found significantly worse cognitive performance in the diabetic participants at baseline [26, 29]. All participants were studied prospectively one to four times, the follow-up time ranging from 2 to 6 years. In all analyses, only participants who survived the interval until the last follow-up were included. The prospective analyses in all five studies showed a significantly greater decline in the diabetic participants in some but not all of the neuropsychological test scores compared with non-diabetic participants.

These findings are dissimilar to the findings of the present study. A possible explanation is that the participants from previous studies were generally younger at study entry. Moreover, baseline cognitive function was better (e.g. mean MMSE score 27.7 [24] or 29.0 [27] vs 25.8 in the present study), and the rate of cognitive decline, even in the non-diabetic group, was much lower (approximately 0.2 MMSE points per year $[24,27]$ vs 0.8 in the present study). The additional rate of cognitive decline that was attributed to diabetes in, for example, the MMSE score in previous studies was 0.05 [27] or 0.25 [24] points per year. The much higher rate of cognitive decline in both diabetic and non-diabetic participants observed in the present study, due to the advanced age of the population involved, may have masked any additional effects of diabetes, leading to a ceiling effect. An alternative hypothesis would be that individuals who reach the age of 85 , despite having diabetes, are survivors who are relatively less susceptible to the adverse effects of this condition. However, this is not supported by the higher mortality rate in the diabetic patients in our study.

Analogous to the present observations, a non-linear relationship with age has been described for other risk factors for cognitive decline and dementia [11, 31-34]. While hypertension, obesity and dyslipidaemia are established risk factors for cognitive dysfunction and dementia in the younger old, these factors may actually be protective in the oldest old. An age-dependent relationship between blood pressure and cognitive function and dementia has been described, whereby high blood pressure in middle age leads to more vascular comorbidities in late life, but there is less evidence for the same negative effect on cognition in later life [11]. Particularly in the oldest old, low blood pressure actually appears to be harmful for cognitive function [11]. Similarly, hypercholesterolaemia and increased BMI are associated with accelerated cognitive decline up to the age of $75[31,32]$. In the oldest old, however, hypercholesterolaemia was found to be associated with a reduced risk of dementia, cognitive decline and death over a 6-year period [33], and low BMI with an increased risk of Alzheimer's disease [34].

It may thus be hypothesised that there is a bell-shaped relationship between age, diabetes and cognitive function. From mid-life onwards the effect of diabetes on cognitive function increases with age; the effect is greatest in the 6575 age range and declines thereafter. Whether this is due to ceiling effects, selective survival or even protective effects of high glucose levels in the oldest old remains to be determined.

The determinants of cognitive impairments in diabetes are unclear. Associations with hyperglycaemia, hyperinsulinaemia [5], hypertension [35], dyslipidaemia and cerebrovascular disease have been found in some but not all studies $[5,6]$. In our study, worse cognitive performance in the diabetes group was associated with macrovascular disease. However, this association did not appear to be specific for diabetes [19] and adjustment for macrovascular disease in the analyses had only a modest effect on the difference in cognitive performance between diabetic and non-diabetic participants. While hypertension has been suggested to be an important determinant of impaired cognition in diabetes [4], in the present study no clear relationship was found between hypertension and cognitive function within the group of diabetes patients, in line with other recent studies $[27,29]$. A higher $\mathrm{HbA}_{1 \mathrm{c}}$ level was largely unrelated to cognitive dysfunction, except for a modest positive effect on the memory tests. This suggests that in the oldest old macrovascular disease is the strongest determinant of cognitive dysfunction in patients with diabetes. However, 
on the basis of the present data we are unable to identify the specific determinant that accounts for the difference in cognitive performance between diabetic and non-diabetic participants.

The strengths of the present study are the population-based sample of the oldest old with a high incidence of cognitive decline, the annual repeated assessments of various cognitive domains, the quantitative assessment of vascular disease at baseline, and the use of linear mixed models that allow optimal use of the data. A limitation of the study is that the classification of diabetes was based on non-fasting blood glucose levels, medical history and medication use, instead of fasting glucose levels or a glucose tolerance test. If anything, this would lead to underestimation of the effect of diabetes on cognitive function. Another limitation is that the diabetic patients showed a higher mortality rate, which results in a relative survival bias for nondiabetic participants. We used linear mixed models, which are best suited to handling this attrition.

In sum, our study shows that very old diabetes patients have lower cognitive function relative to non-diabetic persons at age 85 , but they do not decline faster from age 85 to age 90. Macrovascular disease is associated with lower cognitive performance in diabetes patients of this age group. These findings indicate that the association between diabetes and accelerated cognitive decline that has been documented previously in patients up to 75 years of age is less evident in the oldest old. This suggests that the damage occurs in previous years and that therapies aimed at preventing cognitive decline and dementia should focus on the younger old.

Acknowledgements This study was funded by unrestricted grants from the Netherlands Organisation of Scientific Research (ZonMw) and the Ministry of Health, Welfare and Sports. The research of G. J. Biessels and E. van den Berg was supported by grant 2003.01.004 of the Dutch Diabetes Research Foundation.

\section{References}

1. Wild S, Roglic G, Green A, Sicree R, King H (2004) Global prevalence of diabetes: estimates for the year 2000 and projections for 2030. Diabetes Care 27:1047-1053

2. American Diabetes Association (2002) Standards of medical care for patients with diabetes mellitus. Diabetes Care 25:213-229

3. Awad N, Gagnon M, Messier C (2004) The relationship between impaired glucose tolerance, type 2 diabetes, and cognitive function. J Clin Exp Neuropsychol 26:1044-1080

4. Stewart R, Liolitsa D (1999) Type 2 diabetes mellitus, cognitive impairment and dementia. Diabet Med 16:93-112

5. Strachan MWJ, Deary IJ, Ewing FME, Frier BM (1997) Is type II diabetes associated with an increased risk of cognitive dysfunction? A critical review of published studies. Diabetes Care 20:438-445

6. Allen KV, Frier BM, Strachan MWJ (2004) The relationship between type 2 diabetes and cognitive dysfunction: longitudinal studies and their methodological limitations. Eur $\mathrm{J}$ Pharmacol 490:169-175

7. Cukierman T, Gerstein HC, Williamson JD (2005) Cognitive decline and dementia in diabetes-systematic overview of prospective observational studies. Diabetologia 48:2460-2469

8. Peila R, Rodriguez BL, Launer LJ (2002) Type 2 diabetes, APOE gene, and the risk for dementia and related pathologies: the Honolulu-Asia Aging Study. Diabetes 51:1256-1262

9. Ott A, Stolk RP, Van Harskamp F, Pols HA, Hofman A, Breteler MM (1999) Diabetes mellitus and the risk of dementia: the Rotterdam Study. Neurology 53:1937-1942

10. Ryan CM, Geckle M (2000) Why is learning and memory dysfunction in type 2 diabetes limited to older adults? Diabetes Metab Res Rev 16:308-315

11. Qiu C, Winblad B, Fratiglioni L (2005) The age-dependent relation of blood pressure to cognitive function and dementia. Lancet Neurol 4:487-499

12. der Wiel AB, van Exel E, de Craen AJ et al (2002) A high response is not essential to prevent selection bias: results from the Leiden 85-plus Study. J Clin Epidemiol 55:1119-1125

13. Folstein MF, Folstein SE, McHugh PR (1975) 'Mini-mental state'. A practical method for grading the cognitive state of patients for the clinician. J Psychiatr Res 12:189-198

14. Houx PJ, Shepherd J, Blauw GJ et al (2002) Testing cognitive function in elderly populations: the PROSPER study. PROspective Study of Pravastatin in the Elderly at Risk. J Neurol Neurosurg Psychiatry 73:385-389

15. Yesavage JA, Brink TL, Rose TL et al (1982) Development and validation of a geriatric depression screening scale: a preliminary report. J Psychiatr Res 17:37-49

16. de Craen AJ, Heeren TJ, Gussekloo J (2003) Accuracy of the 15item geriatric depression scale (GDS-15) in a community sample of the oldest old. Int J Geriatr Psychiatry 18:63-66

17. MacFarlane PW, Latif S (1996) Automated serial ECG comparison based on the Minnesota code. J Electrocardiol 29 (Suppl):29-34

18. Gueorguieva R, Krystal JH (2004) Move over ANOVA: progress in analyzing repeated-measures data and its reflection in papers published in the Archives of General Psychiatry. Arch Gen Psychiatry 61:310-317

19. Vinkers DJ, Stek ML, van der Mast RC et al (2005) Generalized atherosclerosis, cognitive decline, and depressive symptoms in old age. Neurology 65:107-112

20. Robertson-Tchabo EA, Arenberg D, Tobin JD, Plotz JB (1986) A longitudinal study of cognitive performance in noninsulin dependent (Type II) diabetic men. Exp Gerontol 21:459-467

21. Hassing LB, Johansson B, Nilsson SE et al (2002) Diabetes mellitus is a risk factor for vascular dementia, but not for Alzheimer's disease: a population-based study of the oldest old. Int Psychogeriatr 14:239-248

22. Brayne C, Gill C, Huppert FA et al (1998) Vascular risks and incident dementia: results from a cohort study of the very old. Dement Geriatr Cogn Disord 9:175-180

23. Xu WL, Qiu CX, Wahlin A, Winblad B, Fratiglioni L (2004) Diabetes mellitus and risk of dementia in the Kungsholmen project: a 6-year follow-up study. Neurology 63:1181-1186

24. Hassing LB, Grant MD, Hofer SM et al (2004) Type 2 diabetes mellitus contributes to cognitive decline in old age: a longitudinal population-based study. J Int Neuropsychol Soc 10:599-607

25. Kanaya AM, Barrett-Connor E, Gildengorin G, Yaffe K (2004) Change in cognitive function by glucose tolerance status in older adults: a 4-year prospective study of the Rancho Bernardo study cohort. Arch Intern Med 164:1327-1333

26. Logroscino G, Kang JH, Grodstein F (2004) Prospective study of type 2 diabetes and cognitive decline in women aged 70 81 years. BMJ 328:548 
27. Fontbonne A, Berr C, Ducimetiere P, Alperovitch A (2001) Changes in cognitive abilities over a 4-year period are unfavorably affected in elderly diabetic subjects: results of the Epidemiology of Vascular Aging Study. Diabetes Care 24:366-370

28. Knopman D, Boland LL, Mosley T et al (2001) Cardiovascular risk factors and cognitive decline in middle-aged adults. Neurology $56: 42-48$

29. Gregg EW, Yaffe K, Cauley JA et al (2000) Is diabetes associated with cognitive impairment and cognitive decline among older women? Study of Osteoporotic Fractures Research Group. Arch Intern Med 160:174-180

30. Haan MN, Shemanski L, Jagust WJ, Manolio TA, Kuller L (1999) The role of APOE epsilon4 in modulating effects of other risk factors for cognitive decline in elderly persons. JAMA 282:40-46
31. Whitmer RA, Gunderson EP, Barrett-Connor E, Quesenberry CP Jr, Yaffe K (2005) Obesity in middle age and future risk of dementia: a 27 year longitudinal population based study. BMJ 330:1360

32. Yaffe K, Barrett-Connor E, Lin F, Grady D (2002) Serum lipoprotein levels, statin use, and cognitive function in older women. Arch Neurol 59:378-384

33. Piguet O, Grayson DA, Creasey $\mathrm{H}$ et al (2003) Vascular risk factors, cognition and dementia incidence over 6 years in the Sydney Older Persons Study. Neuroepidemiology 22:165-171

34. Buchman AS, Wilson RS, Bienias JL, Shah RC, Evans DA, Bennett DA (2005) Change in body mass index and risk of incident Alzheimer disease. Neurology 65:892-897

35. Elias PK, Elias MF, D'Agostino RB et al (1997) NIDDM and blood pressure as risk factors for poor cognitive performance. The Framingham Study. Diabetes Care 20:1388-1395 